\title{
FMR Evidence of Stable Ferromagnetic Correlations at Zigzag Edge States in Graphene
}

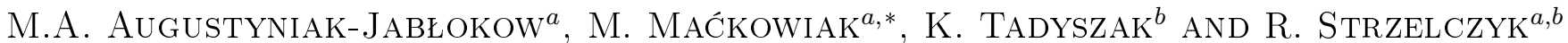 \\ ${ }^{a}$ Institute of Molecular Physics, Polish Academy of Sciences, M. Smoluchowskiego 17, 60-179 Poznań, Poland \\ ${ }^{b}$ NanoBioMedical Centre, Adam Mickiewicz University, Umultowska 8, 61-614 Poznań, Poland
}

\begin{abstract}
We studied magnetic properties of a composite of paraffin and graphene flakes. Magnetic properties of this composite were investigated by ferromagnetic resonance/electron spin resonance technique. The ferromagnetic resonance signal from graphene suspension in paraffin is broad and shifted from magnetic field corresponding to $g \approx 2$ to the low magnetic field. The temperature dependence of the spectra shows that magnetic ordering in the studied system is sensitive to thermal fluctuations. These features are expected for the 1D ferromagnetism and the obtained results are analyzed in the frame of the theoretical predictions concerning magnetic correlations at graphene edges. Therefore, based on our investigation we can conclude the existence of the magnetic edges in graphene and presence of the ferromagnetic correlations between edge spins.
\end{abstract}

DOI: $10.12693 /$ APhysPolA.127.537

PACS: $76.50 .+\mathrm{g}, 68.65 . \mathrm{Pq}$

\section{Introduction}

Graphene, a two-dimensional form of carbon, has attracted considerable attention due to its unique physical properties and potential technological applications. The boundaries of the graphene flake are formed by a combination of armchair and zigzag edges, has spinpolarized nonbonding $\pi$-electron state (edge-state) localized in the zigzag-edge region in spite of the absence of such state in armchair edges, as suggested by theoretical predictions and scanning tunneling microscope/scanning tunneling spectroscopy (STS) experiments [1-4].

According to theoretical studies [5], the edge-state spins are strongly coupled in parallel with each other in a zigzag edge through strong ferromagnetic interaction $J_{0}$ having a strength of $\approx 10^{3} \mathrm{~K}$. In a nanographene sheet with zigzag-edge regions separated by the presence of armchair edges, the ferromagnetically coupled edgestate spins in a zigzag edge are interacting with those in other zigzag edge through interzigzag-edge ferromagnetic/antiferromagnetic interaction $J_{1}$ having a strength of $\approx\left(10^{-1}-10^{-2}\right) J_{0}$ and mediated by conduction $\pi$-carriers.

There have been a large number of theoretical efforts investigating edge state magnetism [6-8] in graphene nanostructures terminated by zigzag edges. However, we are not aware of any direct experimental observation of these phenomena in single-layer graphene. The intrinsic magnetic properties of graphene in finite sizes are far from being understood, because magnetic signal from one piece of graphene in finite sizes is too weak to be detected by macro-magnetic measurement. Therefore, a massive amount of high-purity graphene (at least in milligramscale) is an essential prerequisite to unveil the intrinsic

* corresponding author; e-mail: mackow@ifmpan.poznan.pl magnetism displayed by graphene edges. Even the possibilities of such phenomena were questioned by the fact that no true long-range magnetic ordering in 1D systems is possible at finite temperatures [9]. Nevertheless, nanometer range spin correlation lengths in certain 1D systems have been achieved in practice [10]. Establishing the range of magnetic order at graphene edges as well as the underlying physical mechanisms is thus crucial for understanding and practical realization of the nanoelectronic and spintronic devices.

In this work we study the magnetic correlations at zigzag edges of single-layer graphene by ferromagnetic resonance/electron spin resonance (FMR/ESR) measurements. In particular, the use of FMR is noticeable. The ferromagnetically coupled edge spins gave a broad FMR signal shifted to the low magnetic field. One of the advantages of FMR over conventional magnetization measurements is that it yields information on the dynamics of the system. A mixture of microscopic phases containing a ferromagnetic contribution and a paramagnetic one is not easy to discriminate in a DC magnetization measurement, while it should be clearly distinguished in an FMR experiment. The zigzag edges exhibit ferromagnetic behavior when their size is sufficiently large, wherein the internal field undergoes ferromagnetic ordering revealing FMR lines. We observed the FMR lines in single-layer graphene, which provide the most direct experimental proof of the theoretical prediction of ferromagnetic correlations at zigzag edge states so far.

\section{Material and methods}

The studied material was reduced graphene oxide ( $\mathrm{rGO}$ ), obtained by the thermal reduction of graphene oxide (GO) in liquid paraffin. After reduction rGO flakes were subjected to ultrasound cavitation, a process which causes breakage of graphene flakes and uncovers new edges. The used GO was obtained from graphite flakes by a Hummers method [11]. As the GO was single-layer 


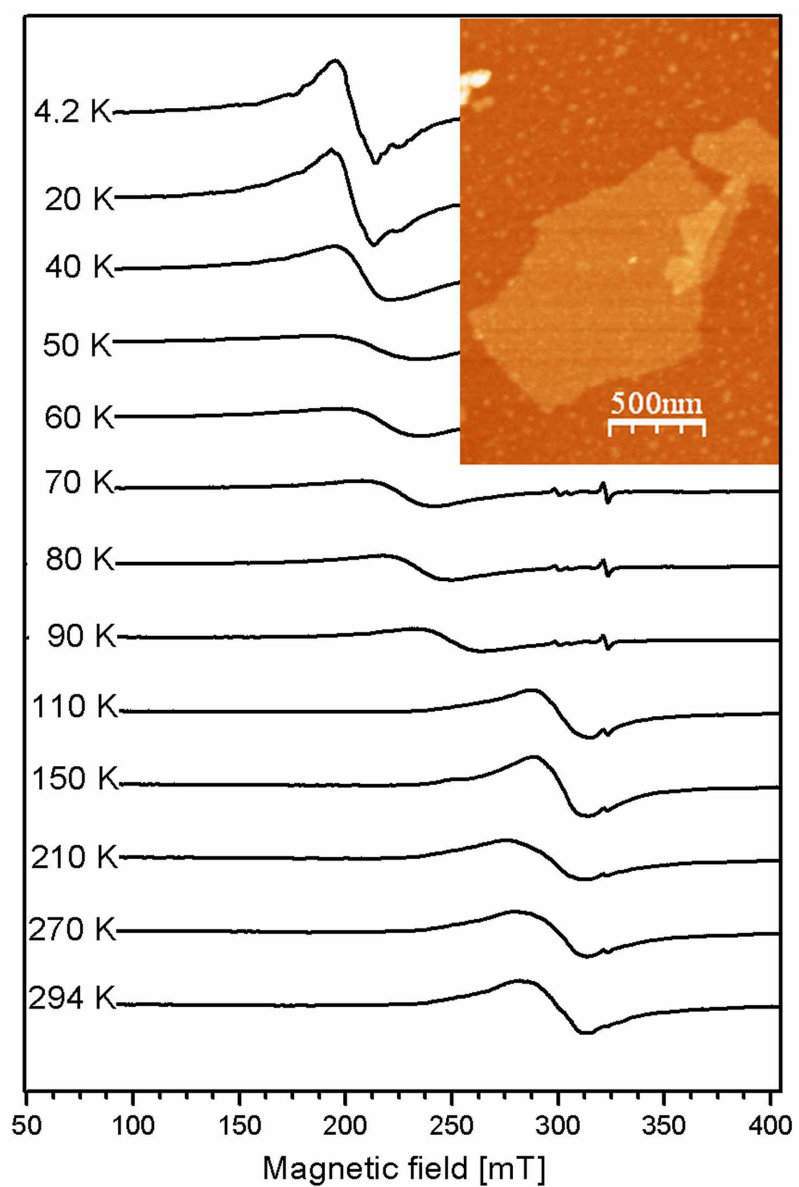

Fig. 1. Temperature variation of the FMR spectra in the ensemble of single-layer graphene flakes in paraffin. The inset shows AFM image of GO flake. The low intense high field signals are due to impurities generated in the paraffin during GO thermal reduction.

(inset in Fig. 1), the rGO in the paraffin matrix is also single-layer.

ESR measurements were performed on the ensemble of graphene flakes with use of X-band RADIOPAN SX spectrometer with Oxford CF935 cryostat allowing the measurements in the temperature range of $4.2-300 \mathrm{~K}$.

\section{Results and discussion}

The FMR signal from graphene suspension in paraffin is broad and is shifted from magnetic field corresponding to $g \approx 2$. It is stable only for the low graphene concentration. For higher concentrations, obtained, e.g., by evaporation of paraffin, the signal disappears after several hours (presumably due to coupling between graphene flakes). This instability of the signal is, paradoxically, the proof that the observed signal is related to the graphene and not due to the presence of ferromagnetic impurities.

Temperature variation of the FMR/ESR spectra of the field cooled $(700 \mathrm{mT})$ single-layer graphene is shown in Fig. 1. The presence of a distinct FMR lines situated at ca. $200 \mathrm{mT}$ with a peak-to-peak width of $c a .20 \mathrm{mT}$ is evident below $T=50 \mathrm{~K}$. The unexpected, for the powder-like sample, shift of the signal to the low field can be a sign of the 1D ferromagnetism. Magnetization of the ferromagnetically ordered spins of the monoatomic Co chains deposited on $\mathrm{Pt}$ substrate aligns in the easy axis direction [10]. Similarly shifted signal was observed also for the ultrasound exfoliated $\mathrm{MoS}_{2}$ [12]. Presence of ferromagnetism in this material was confirmed by magnetization measurements.

Above $50 \mathrm{~K}$ the intensity of the FMR lines drops down and their resonance field shifts up indicating the lowering of the local field. Above $100 \mathrm{~K}$ the wide FMR lines characterized by low values of widely distributed effective local fields are visible. To understand this behavior we have to analyze the magnetic spin correlation length, which is responsible for magnetic ordering in ferromagnetic nanodomains formed by zigzag edges.

In the ferromagnetic materials the resonant microwave absorption for a given frequency is controlled by an effective field $B_{\text {eff }}$, which is a vector sum of the applied external field and various contributions of the internal magnetization. The localized edge-state spins in graphene contribute to the formation of an unconventional carbon-based magnetic system, as the edge state is strongly spin polarized. The resonance center field $\left(B_{\text {center }}\right)$ for the FMR signals shifts to a lower value (about $200 \mathrm{mT}$ ). It can be understood from the condition for resonance in the presence of local magnetic field $\left(B_{A}\right): h f / \mu_{\mathrm{B}} g=B_{\text {center }}+B_{A}$, where $h$ is the Planck constant, $g \approx 2$ for a free electron, $f(8.970 \mathrm{GHz})$ is the fixed frequency of the applied microwave magnetic field, and $\mu_{\mathrm{B}}$ is the Bohr magneton, respectively. Magnetic order in low-dimensional systems is exceptionally sensible to thermal fluctuations. In particular, the Mermin-Wagner theorem excludes long range order in one-dimensional magnetic systems (such as the magnetic graphene edges) at any finite temperature [9]. Nevertheless, a recent theoretical report using quantum Monte Carlo supports the existence of such a long spin-spin correlation length along the zigzag edge and justifies the picture obtained from the one-electron theories [13]. One of the most appealing experimental examples of 1D magnetism is monoatomic Co chains on Pt substrate characterized by a ferromag-

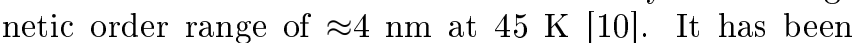
shown that at low temperature $(T=10 \mathrm{~K})$ the whole system becomes ferromagnetic (the magnetization of each spin-block aligns in the easy axis direction). At higher temperature $(T=45 \mathrm{~K})$ the linear monoatomic chain spontaneously breaks down into segments with different orientation of the magnetization due to thermal fluctuations [10]. The range of magnetic order is limited by the temperature-dependent spin correlation lengths.

The mechanism of the transverse and longitudinal spin excitations have been explored using density functional theory (DFT) calculations [8]. In graphene the magnetic correlation length at $T=50 \mathrm{~K}$ is estimated to only $5 \mathrm{~nm}$ (that corresponds to a linear zigzag chain containing about 20 spins) [8]. As shown in Fig. 2, the local 
field determining the FMR frequency is practically constant up to $T=50 \mathrm{~K}$. The local field is created mainly by the nearest neighbor spins in the quasi-1D ferromagnetic nanodomains formed by zigzag chains. As far as the rapidly decreasing with temperature magnetic correlation lengths are longer than the ferromagnetic nanodomains, the local effective magnetic moment is constant. Above $50 \mathrm{~K}$ the magnetic correlations become too short to produce stable local field and fast magnetic spin fluctuations lead to averaging of the effective field.

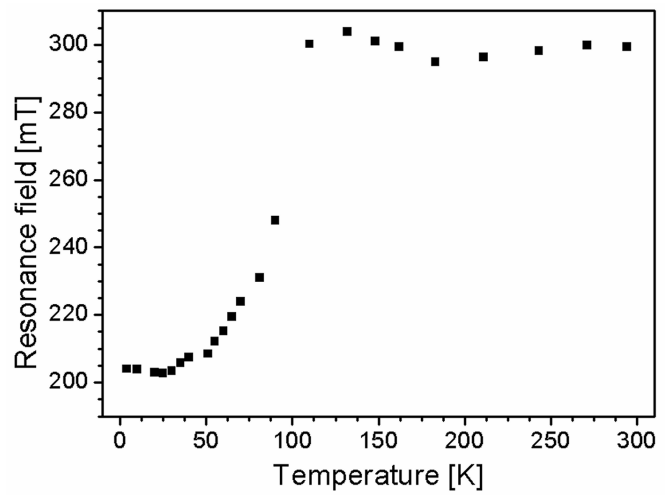

Fig. 2. Temperature dependence of the resonance field of the FMR line for $\nu=9 \mathrm{GHz}$.

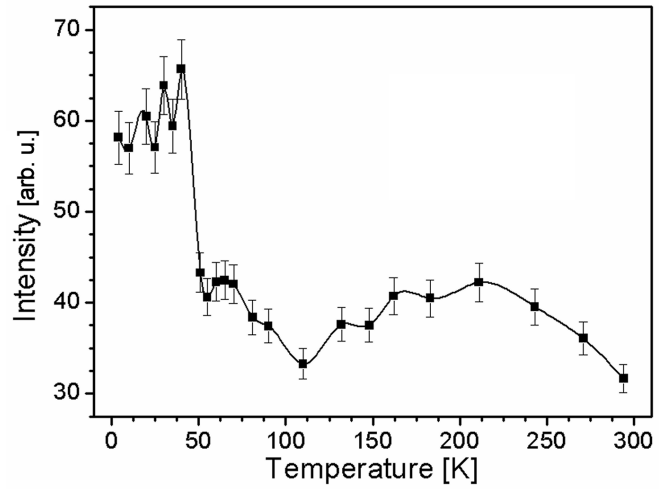

Fig. 3. Temperature dependence of the doubleintegrated intensity of the first-derivative FMR line.

Figure 3 shows temperature dependence of the doubleintegrated intensity of the first-derivative FMR line. This value is proportional to the magnetization. As shown in Fig. 3, the rapid decrease of the magnetization is a clear evidence of breaking down of the long-range ferromagnetic order. As predicted by the theory [8], the linear ferromagnetic nanodomain breaks down into spin segments due to thermal fluctuations (the magnetic correlation length decreases). The presence of wide spectral lines at temperatures above $100 \mathrm{~K}$, characterized by low values of widely distributed effective local fields, is a consequence of the development of spin glass state [4]. In general, a spin glass state develops when the strengths of exchange interaction vary randomly in space. At this temperature range the magnetic correlation lengths are very short and rapid fluctuations of a frustrated spin system take place. Bhowmick and Shanoy [6] calculated that the zigzag edges of single layer graphene nanostructures that are longer than three to four repeat units are always magnetic, irrespective of other regular or irregular edges.

\section{Conclusions}

In this study we provided the direct experimental evidence of ferromagnetic ordering in the zigzag edges of graphene. Based on our investigation we can conclude the existence of the magnetic edges in graphene and presence of the ferromagnetic correlations between edge spins. Magnetic ordering is determined by the spin correlation length. Magnetic order in low-dimensional systems is exceptionally sensitive to thermal fluctuations.

The FMR technique requires much smaller sample comparing to conventional magnetometric measurements, and opens new possibilities for investigations of these issues in carbon-based magnetic nanostructures.

\section{References}

[1] V.L.J. Joly, K. Takahara, K. Takai, K. Sugihara, T. Enoki, M. Koshino, H. Tanaka, Phys. Rev. B 81, 245428 (2010).

[2] X. Jia, J. Campos-Delgado, M. Terrones, V. Meuniere, M.S. Dresselhaus, Nanoscale 3, 86 (2011).

[3] M. Acik, Y.J. Chabal, Jpn. J. Appl. Phys. 50, 070101 (2011).

[4] T. Enoki, Phys. Scr. T 146, 014008 (2012).

[5] Y.W. Son, M.L. Cohen, S.G. Louie, Nature 444, 347 (2006).

[6] S. Bhowmick, V.B. Shenoy, J. Chem. Phys. 128, 244717 (2008).

[7] W.L. Wang, S. Meng, E. Kaxiras, Nano Lett. 8, 241 (2008).

[8] O.V. Yazyev, M.I. Katsnelson, Phys. Rev. Lett. 100 047209 (2008).

[9] N.D. Mermin, H. Wagner, Phys. Rev. Lett. 17, 1133 (1966).

[10] P. Gambardella, A. Dallmeyer, K. Maiti, M.C. Malagoli, W. Eberhardt, K. Kern, C. Carbone, Nature 416, 301 (2002).

[11] W.S. Hummers, R.E. Offeman, J. Am. Chem. Soc. 80, 1339 (1958).

[12] D. Gao, M. Si, J. Li, J. Hang, Z. Zhang, Z. Yang, D. Xue, Nanoscale Res. Lett. 8, 129 (2013).

[13] H. Feldner, Z.Y. Meng, T.C. Lang, F.F. Assaad, S. Wessel, A. Honecker, Phys. Rev. Lett. 106 , 226401 (2011). 